BEZERRA NETO F; GOMES EG; OLIVEIRA AM. 2007. Produtividade biológica em sistemas consorciados de cenoura e alface avaliada através de indicadores agroeconômicos e métodos multicritério. Horticultura Brasileira 25: 193-198.

\title{
Produtividade biológica em sistemas consorciados de cenoura e alface avaliada através de indicadores agroeconômicos e métodos multicritério
}

\author{
Francisco Bezerra Neto ${ }^{1}$; Eliane G. Gomes ${ }^{2}$; Antônio Marcos de Oliveira ${ }^{1}$ \\ 1UFERSA, Programa Pós-Graduação em Fitotecnia, C. Postal 137, 59625-900 Mossoró-RN; ²Embrapa, SGE, Parque Estação Biológica, \\ Av. W3 Norte Final, 70770-901 Brasília-DF; *Bolsistas CNPq; bezerra@ufersa.edu.br; eliane.gomes@embrapa.br
}

\begin{abstract}
RESUMO
O experimento foi conduzido de maio a setembro de 2002, na UFERSA, Mossoró-RN, para avaliar consórcios de cenoura e alface, através de critérios agronômicos, econômicos (rendimentos financeiros) e de eficiência produtiva (avaliada segundo modelos de Análise de Envoltória de Dados - DEA). Para agregar a informação de todos os critérios e identificar os melhores tratamentos, foram utilizados os métodos multicritério ordinais de Borda, Condorcet e Copeland. O delineamento experimental foi blocos casualizados, em fatorial 3 (cultivo solteiro e consorciado, em faixas, com três fileiras de cenoura e três de alface $(3 \mathrm{~F})$ e quatro de cenoura e quatro de alface (4F)) x 4 (cultivares de alface americana: Lucy Brown, Tainá, Laurel e Mesa 659), com quatro repetições. Em cada bloco foi plantada a cenoura solteira. Avaliou-se o índice de uso eficiente da terra (UET), o índice de lucratividade (IL) e a eficiência produtiva DEA. Uma análise de variância univariada foi usada para avaliar estes indicadores, no esquema fatorial $2 \times 4+1$ (dois sistemas consorciados em faixas, quatro cultivares de alface e cenoura solteira). Os UETs dos sistemas consorciados foram significativamente superiores ao UET do cultivo solteiro, com a vantagem dos sistemas consorciados variando de 90 (cenoura + cultivar Tainá 4F) a 133\% (cenoura + cultivar Mesa 659 4F). Não se observou diferença significativa no índice de lucratividade entre os cultivos solteiro e consorciado. $\mathrm{Na}$ eficiência produtiva DEA foi observada interação significativa entre sistemas de cultivo e cultivares de alface. Os métodos multicritério indicaram Cenoura + Mesa 659 (4F), Cenoura + Laurel (3F) e Cenoura + Mesa 659 (3F) como os melhores sistemas de cultivo. A eficiência produtiva DEA e a ordenação dos métodos multicritério foram mais eficientes na discriminação do desempenho dos sistemas de cultivo consorciados que os indicadores agroeconômicos.
\end{abstract}

Palavras-chave: Daucus carota, Lactuca sativa, eficiência de sistemas consorciados, análise de envoltória de dados, métodos multicritério ordinais.

\begin{abstract}
Biological productivity in carrot and lettuce intercropping systems assessed by means of agroeconomic indicators and multicriteria methods
\end{abstract}

The experiment was carried out from May to September 2002, at the Federal University of the Semi-Arid Tropic in Mossoró-RN, to evaluate the performance of carrot-lettuce intercropping systems by means of agronomic criteria, economic (profit margin) indicators, and yield efficiency (assessed through Data Envelopment Analysis models - DEA). We also used the ordinal multicriteria methods of Borda, Condorcet, and Copeland to aggregate information to all criteria and to identify the best treatments. The experimental design was randomized complete blocks, in a 3 (sole crop, three carrot rows alternate with three lettuce rows $-3 \mathrm{~F}$, and four carrot rows alternate with four lettuce rows - 4F) x 4 (crisp head lettuce cultivars: Lucy Brown, Tainá, Laurel, and Mesa 659) factorial scheme, with four replications. In each block, carrot was grown as sole crop. Agroeconomic indicators, such as land equivalent ratio - LER - and profit margin, yield efficiency of intercropping systems by DEA models, were assessed. A univariate analysis of variance in a $2 \times 4+$ 1 factorial scheme (treatments from two intercropping systems versus four crisp head lettuce cultivars, plus carrot in sole crop) was used to assess all indicators. LER of intercropping systems were significantly higher as compared to that of sole crop, with the efficiency of intercropping systems varying from 90 (Carrot + Tainá - 4F) to 133\% (Carrot + Mesa $659-4 \mathrm{~F}$ ). There was no significant difference in the profit margin between sole crop and intercropping systems. A significant interaction was observed between cropping systems and lettuce cultivars for yield efficiency by DEA. According to the ranks of ordinal multicriteria methods, the best intercropping systems were carrot + Mesa 659 (4F), carrot + Laurel (3R) and carrot + Mesa 659 (4R). The yield efficiency by DEA and the ordering of ranks based on ordinal multicriteria methods were more efficient than the agroeconomic indicators for discriminating the intercropping systems performance.

Keywords: Daucus carota, Lactuca sativa, intercropping efficiencies, Data Envelopment Analysis, ordinal multicriteria methods.

(Recebido para publicação em 10 de outubro de 2005; aceito em 12 de abril de 2007)

$\mathrm{D}$ eve-se reconhecer que na avaliação de experimentos consorciados não há uma forma simples de análise estatística, que seja apropriada a todas as formas de dados de consorciação. Mesmo para um simples conjunto de dados experimentais será importante usar di- ferentes formas de análise, devido ao fato de as diferentes culturas componentes de um sistema consorciado terem dados que podem ocorrer em diferentes formas estruturais. Estas estruturas dos dados são complexas, com diferentes formas de informação sobre o rendimen- to disponível para diferentes subconjuntos de unidades experimentais (Mead, 1990).

Um dos métodos de análise de dados é o de formar um índice de rendimento combinado para cada parcela consorciada e então, analisar a variável 
resultante destes rendimentos combinados. O índice de uso eficiente da terra (UET) tem sido utilizado neste tipo de análise para medir a eficiência biológica de um sistema consorciado (Riley, 1984). Porém, os sistemas consorciados devem ser julgados não só por meio de indicadores agronômicos, como a UET, mas também por indicadores econômicos, como rendas bruta e líquida e índice de lucratividade (Beltrão et al., 1984; Anandajayasekeram, 1990). Uma alternativa para combinar os rendimentos advindos de um experimento sobre consorciação é considerar a produção equivalente. Esta nova variável pode basear-se em diversas características como, por exemplo, quantidade total de proteínas e valor econômico. Em Gomes $\&$ Souza (2005), foi proposta uma nova abordagem para agregar em um índice unidimensional tratamentos em situação experimental com resposta multidimensional, como o caso de consórcios. Os autores usaram modelos de Análise de Envoltória de Dados (Data Envelopment Analysis - DEA) para esse fim, com o cálculo de uma medida que pode ser chamada de "eficiência produtiva".

Modelos DEA têm como objetivo calcular a eficiência relativa de unidades produtivas, conhecidas na literatura como unidades de tomada de decisão ou DMUs (Decision Making Units). Usamse em sua formulação problemas de programação linear (PPLs) que otimizam cada observação individual de modo a estimar a fronteira eficiente, linear por partes, composta das unidades que apresentam as melhores práticas dentro da amostra em avaliação (unidades eficientes). A definição de eficiência é baseada na relação entre os resultados obtidos e os recursos empregados por cada entidade em avaliação (DMU). A vantagem da técnica DEA frente a outros modelos de produção é a possibilidade de incorporar insumos (inputs) múltiplos e produtos (outputs) múltiplos no cálculo de medida de eficiência única.

Ainda assim, os agrossistemas consorciados não podem ser avaliados adequadamente somente pelos critérios de produção ou do valor econômico. Avaliar diferentes alternativas sob a ótica de múltiplos critérios é um dos objetivos dos métodos do Apoio Multicritério à
Decisão. Dentre esses métodos estão os chamados métodos ordinais, considerados bastante intuitivos. Eles são ainda pouco exigentes em termos computacionais e em relação às informações necessárias por parte dos decisores. Nessa classe de métodos estão os métodos de Borda, Condorcet e Copeland (Barba-Romero \& Pomerol, 1997).

O objetivo deste trabalho foi avaliar consórcios de cenoura e alface, produzidos na região oeste do estado do Rio Grande do Norte, através de critérios agronômicos (métodos de UET), econômicos (rendimentos financeiros) e de "eficiência produtiva" (avaliadas segundo modelos DEA). Para "agregar" a informação de todos os critérios e identificar os melhores tratamentos, foram usados os métodos multicritério ordinais de Borda, Condorcet e Copeland.

\section{MATERIAL E MÉTODOS}

O experimento foi realizado na horta da UFERSA, de junho a setembro de 2002, em solo classificado como Argissolo Vermelho-Amarelo Eutrófico (Embrapa, 1999). As características do solo da área experimental são: $\mathrm{pH}$ (água $1: 2,5)=7,55 ; \mathrm{Ca}=7,12 \mathrm{cmol}_{\mathrm{c}} \mathrm{dm}^{-3} ; \mathrm{Mg}$ $=4,02 \mathrm{cmol}_{\mathrm{c}} \mathrm{dm}^{-3} ; \mathrm{K}=0,60 \mathrm{cmol}_{\mathrm{c}} \mathrm{dm}^{-3}$; $\mathrm{Na}=0,25 \mathrm{cmol}_{\mathrm{c}} \mathrm{dm}^{-3} ; \mathrm{Al}=0,00 \mathrm{cmol}_{\mathrm{c}}$ $\mathrm{dm}^{-3}$ e $\mathrm{P}=554 \mathrm{mg} \mathrm{dm}^{-3}$. O delineamento experimental utilizado foi blocos casualizados completos, em esquema fatorial 3 x 4, com quatro repetições. Os tratamentos resultaram da combinação de três sistemas de cultivos consorciados (solteiro, consorciado em faixas com três fileiras de cenoura alternadas com três fileiras de alface e consorciado em faixas com quatro fileiras de cenoura alternadas com quatro fileiras de alface), com quatro cultivares de alface tipo americana (Lucy Brown, Tainá, Laurel e Mesa 659). Em cada bloco foi plantada uma parcela com a cenoura solteira.

Duas análises de variância univariadas foram utilizadas: a primeira para avaliar as características da alface no esquema fatorial $3 \times 4$ (provenientes de três sistemas de cultivo e quatro cultivares de alface) e, a segunda, para avaliar os indicadores agroeconômicos e as características da cenoura no esquema fatorial $2 \times 4+1$ (provenientes da combinação de dois sistemas consorciados em faixas com quatro cultivares de alface, além da cenoura solteira). Neste artigo, o interesse foi analisar os indicadores de eficiência dos sistemas.

No cultivo solteiro da alface, as parcelas tinham uma área total de 1,20 m² $(1,00 \times 1,20 \mathrm{~m})$, com uma área útil de $0,48 \mathrm{~m}^{2}(0,60 \times 0,80 \mathrm{~m})$, contendo doze plantas, no espaçamento de $0,20 \times 0,20$ $\mathrm{m}$. No cultivo da cenoura a área útil foi de $0,60 \mathrm{~m}^{2}(0,60 \times 1,00 \mathrm{~m})$, contendo 30 plantas, no espaçamento de $0,20 \mathrm{x}$ $0,10 \mathrm{~m}$. Nos sistemas consorciados, cada parcela foi constituída de quatro faixas alternadas com três e quatro fileiras cada faixa. As parcelas com faixas de três fileiras tinham uma área total de 2,88 $\mathrm{m}^{2}$ $(2,40 \times 1,20 \mathrm{~m})$ e uma área útil de 1,20 $\mathrm{m}^{2}(1,20 \times 1,00 \mathrm{~m})$, contendo 30 plantas de alface e 60 de cenoura. Nas parcelas com faixas de quatro fileiras, a área total de cada parcela foi de $3,84 \mathrm{~m}^{2}$, com uma área útil de 1,60 m² (1,60 x 1,00 $\mathrm{m})$, contendo 40 plantas de alface e 80 plantas de cenoura. Nestes sistemas em faixas, os espaçamentos da cenoura foram de 0,60 e $0,80 \mathrm{~m}$ entre faixas de três e quatro fileiras, respectivamente e de $0,20 \times 0,05 \mathrm{~m}$ entre fileiras e plantas. $\mathrm{O}$ espaçamento entre faixas de alface foi o mesmo da cenoura e o espaçamento entre fileiras e plantas foi de $0,20 \times 0,10$ $\mathrm{m}$. O espaçamento dentro das fileiras de cada cultura no consórcio foi diferente do cultivo solteiro, para que fosse mantida constante a população em ambos os sistemas. A população recomendada para o cultivo solteiro da alface na região é de 250.000 plantas ha ${ }^{-1}$ (Silva, 1999) e, no cultivo da cenoura, 500.000 plantas ha ${ }^{-1}$ (Siqueira, 1995), sem levar em consideração os $30 \%$ de área de trânsito, composta de corredores e estradas. Mas, para as características de massa seca e produtividade das culturas foram feitas as correções para $70 \%$ da área plantada.

A cultivar de cenoura utilizada foi Brasília, indicada para a região Nordeste (Vieira et al., 1983). As cultivares de alface utilizadas foram do tipo americana (repolhuda crespa): Lucy Brown, Tainá, Laurel e Mesa 659 (Sementes 
Sakama, 2002), que não chegam a formar cabeça nas condições de alta temperatura e luminosidade do Estado do Rio Grande do Norte (Oliveira, 2004). Nos canteiros de plantio foram realizadas adubações com $80 \mathrm{t} \mathrm{ha}^{-1}$ de esterco de bovinos, com $30 \mathrm{~kg} \mathrm{ha}^{-1}$ de nitrogênio e $30 \mathrm{~kg} \mathrm{ha}^{-1}$ de $\mathrm{K}_{2} \mathrm{O}$.

As cultivares de alface foram semeadas em dois cultivos sucessivos, em 20 de maio e 12 de julho de 2002, em copos descartáveis de $150 \mathrm{~mL}$, contendo como substrato uma mistura de vermiculita e húmus, na proporção de 1:2. Foram semeadas três a cinco sementes por recipiente. Sete dias após a emergência (DAE) efetuou-se o primeiro desbaste, deixando-se três plântulas por recipiente; quinze DAE, o segundo desbaste, deixando-se apenas uma plântula por recipiente. As mudas foram produzidas sob sombreamento, utilizando-se uma estufa coberta com tela de nylon de cor branca. A cenoura, cultura principal, foi semeada em 4 de junho de 2002. O primeiro desbaste foi realizado 23 dias após a semeadura (DAS), deixando-se três plântulas por cova, e, o segundo, 35 DAS, deixando-se apenas uma planta por cova.

Nas parcelas solteiras e consorciadas da cenoura form realizadas uma adubação nitrogenada em cobertura, 42 DAS, enquanto nas parcelas de alface, tanto no primeiro como no segundo cultivo, efetuaram-se adubações foliares de $30 \mathrm{~mL} 20 \mathrm{~L}^{-1}$ de água da formulação $14 \%$ de $\mathrm{N}, 4 \%$ de $\mathrm{P}_{2} \mathrm{O}_{5}, 6 \%$ de $\mathrm{K}_{2} 0,0,8 \%$ de $\mathrm{S}, 1,5 \%$ de $\mathrm{Mg}, 2 \%$ de $\mathrm{Zn}, 1,5 \%$ de $\mathrm{Mn}$, $0,1 \%$ de B e $0,05 \%$ de Mo, 28, 35 e 42 DAS. As mudas de alface do primeiro e segundo cultivo foram transplantadas para as faixas adjacentes à cenoura, 24 DAS, quando a cultura da cenoura encontrava-se com 7 e 62 dias da semeadura, respectivamente. Antes da instalação do experimento em campo, foi realizada a solarização dos canteiros de plantio, durante 60 dias, para evitar ou reduzir a população de fitopatógenos do solo que pudesse prejudicar a produtividade da cultura da cenoura. Durante a condução do experimento foram efetuadas capinas manuais e irrigação pelo sistema de micro-aspersão.

A colheita das cultivares de alface do primeiro cultivo foi realizada nos dias 8 e 9 de julho de 2002, estando a cenoura com aproximadamente 35 dias da semeadura e, a alface, com 49 e 50 dias da semeadura. A colheita das cultivares de alface do segundo cultivo foi realizada nos dias 27 e 28 de agosto de 2002, estando a cenoura com aproximadamente 85 dias da semeadura e, a alface, com 47 e 48 dias da semeadura. A colheita da cenoura foi realizada em 2 de setembro de 2002. As características avaliadas na cenoura e na alface foram produtividade comercial (obtida da massa das raízes das plantas da parcela útil, livres de rachaduras, bifurcações, nematóides e danos mecânicos, e expressa em t ha-1) e rendimento de folha (avaliado através da massa fresca da parte aérea de todas as plantas da parcela útil, expressa em $\mathrm{t} \mathrm{ha}^{-1}$ ).

Indicadores agroeconômicos foram utilizados para medir o desempenho dos sistemas consorciados (Beltrão et al., 1984). Entre estes estão o índice de uso eficiente da terra (UET), renda bruta (RB), renda líquida (RL) e índice de lucratividade (IL). O índice UET é dado pela expressão: $\left(\mathrm{I}_{\text {cenoura }} / \mathrm{S}_{\text {cenoura }}\right)+\left(\mathrm{I}_{\text {alface }} /\right.$ $\mathrm{S}_{\text {alface}}$ ), onde I e $\mathrm{S}$ representam as produtividades dos sistemas consorciado e solteiro de cada cultura componente. É definido como a área relativa de terra, sob condições de plantio isolado, que é requerida para proporcionar as produtividades alcançadas no consórcio. A renda bruta (RB) foi obtida multiplicandose a produtividade da cultura em cada tratamento pelo valor do produto pago ao produtor no mês de outubro de 2002, que correspondeu a $\mathrm{R} \$ 1,10 \mathrm{~kg}^{-1}$ para a alface e, $\mathrm{R} \$ 0,65 \mathrm{~kg}^{-1}$, para a cenoura. A renda líquida (RL) foi calculada subtraindo-se da renda bruta, os custos de produção, provenientes de insumos e serviços. Estes custos (C) foram calculados para cada tratamento, baseado nos coeficientes de custo de insumos e serviços utilizados em um hectare de cenoura e alface ao nível experimental. Foram considerados os preços de insumos e serviços vigentes no mês de outubro de 2002, na cidade de Mossoró. $\mathrm{O}$ índice de lucratividade (IL) foi obtido da relação entre a RL e RB e expresso em percentagem.

Além destes indicadores, utilizou-se ainda a eficiência produtiva, calculada com base na abordagem proposta por Gomes \& Souza (2005), que utiliza modelos de Análise de Envoltória de Dados (DEA) para gerar uma medida univariada a partir de uma resposta multivariada. Em modelagem DEA são definidas as unidades a avaliar, as variáveis (insumos e produtos) e o modelo a ser usado. As unidades em avaliação foram os tratamentos, em um total de 36 (provenientes da combinação de dois sistemas de cultivo com quatro cultivares de alface, mais a cenoura solteira, todos com quatro repetições). Os produtos foram os valores ou quantidades das variáveis respostas medidas em cada tratamento, neste caso a produtividade da cenoura, a produtividade da alface no primeiro e no segundo cultivos e a renda líquida do sistema. Para avaliar o desempenho de cada parcela, considerou-se que cada uma utilizou-se de um único insumo com nível unitário (Lovell \& Pastor, 1999; De Koeijer et al., 2002; Soares de Mello \& Gomes, 2004; Gomes \& Souza, 2005; Leta et al., 2005), já que os outputs incorporam os possíveis inputs.

Neste artigo o modelo escolhido foi o DEA CCR (Charnes et al., 1978), já que não há evidências de diferenças de escala significativas e as variáveis disponíveis são, tipicamente, valores ou quantidades produzidas. Nesse modelo, a eficiência relativa da DMU é definida como a razão da soma ponderada das componentes do vetor de produção pela soma ponderada das componentes do vetor de insumo usado no processo de produção. Os pesos utilizados nas ponderações das componentes de insumos e produtos (preços sombra) são distintos e são obtidos da solução dos PPLs que atribuem a cada DMU os pesos que maximizam a sua eficiência, ou seja, são atribuídos da forma mais benevolente a cada DMU.

Em (1) mostra-se a formulação arquimediana do modelo DEA CCR clássico para o caso aqui estudado (Cooper et al., 2000), na qual $y_{j k}$ é o valor do output $j, j=1, \ldots, 4$, para o tratamento $k$, $k=1, \ldots, 36 ; u_{j}$ é o peso atribuído ao output $j, O$ é o tratamento em análise.

$$
\begin{aligned}
& \operatorname{Max} \sum_{j=1}^{4} u_{j} y_{j O} \\
& \text { sujeito a } \\
& \sum_{j=1}^{4} u_{j} y_{j k} \leq 1, \forall k \\
& u_{j} \geq 0, \forall j
\end{aligned}
$$


Tabela 1. Índice de uso eficiente da terra (UET), índice de lucratividade (IL) e eficiência produtiva (índice DEA), em função de sistemas de cultivo e de cultivares de alface americana (Land equivalent ratio (UET), profit margin (IL) and yield efficiency by DEA, as function of cropping systems and crisp head lettuce cultivars). Mossoró, UFERSA, 2002.

\begin{tabular}{lcccc}
\hline Sistemas de cultivo & UET & IL (\%) & \multicolumn{2}{c}{ Eficiência Produtiva DEA } \\
\hline Faixa c/ 3 fileiras & $2,23 \mathrm{a}$ & $69,61 \mathrm{a}$ & \multicolumn{2}{c}{$0,785 \mathrm{~b}$} \\
Faixa c/ 4 fileiras & $2,13 \mathrm{a}$ & $66,93 \mathrm{a}$ & \multicolumn{2}{c}{$0,817 \mathrm{~b}$} \\
Solteiro & $1,00 *$ & $71,66 \mathrm{~ns}$ & \multicolumn{2}{c}{$0,770 *$} \\
\hline \multirow{2}{*}{ Cultivares } & & & \multicolumn{2}{c}{ Sistemas em faixas } \\
\cline { 4 - 6 } & $2,21 \mathrm{a}$ & $67,07 \mathrm{a}$ & $0,752 \mathrm{aA}$ & $0,751 \mathrm{bA}$ \\
Lucy Brown & $2,02 \mathrm{a}$ & $68,60 \mathrm{a}$ & $0,786 \mathrm{aA}$ & $0,663 \mathrm{bA}$ \\
Tainá & $2,16 \mathrm{a}$ & $68,84 \mathrm{a}$ & $0,846 \mathrm{aA}$ & $0,856 \mathrm{abA}$ \\
Laurel & $2,32 \mathrm{a}$ & $68,57 \mathrm{a}$ & $0,756 \mathrm{aA}$ & $0,998 \mathrm{aA}$ \\
Mesa 659 & 18,49 & 6,57 & \multicolumn{2}{c}{14,11} \\
\hline C.V. (\%) & &
\end{tabular}

Médias seguidas de mesma letra minúscula nas colunas ou maiúscula nas linhas não diferem entre si, teste de Tukey, $\mathrm{p}<0,05$ (Means followed by the same small letter in the column or capital letter in the line do not differ from each other, Tukey's test, $\mathrm{p}<0,05$ ).

* Significativamente diferente da média dos sistemas de cultivo consorciados em faixas (Significantly different from the mean for the strip-intercropped systems); ns = Não significativo (Non-significant).

De posse dos valores de cada um desses critérios, uma análise de variância univariada foi utilizada nas variáveis selecionadas. Adicionalmente, para avaliar os diferentes tratamentos segundo todos os critérios considerados, foram utilizados métodos multicritério, que têm como objetivo avaliar diferentes alternativas sob a ótica de múltiplos critérios (Barba-Romero \& Pomerol, 1997). A classe de métodos utilizada foi a dos métodos ordinais, considerados bastante intuitivos e pouco exigentes tanto em termos computacionais, quanto em relação às informações necessárias por parte dos decisores; do decisor não são necessárias mais do que as pré-ordens relativas a cada critério. Neste artigo foram usados os três métodos multicritério ordinais referenciados na literatura, a saber, métodos de Borda, de Condorcet e de Copeland.

Para o uso do método de Borda, o decisor deve ordenar as alternativas de acordo com as suas preferências. A alternativa mais preferida recebe um ponto, a segunda dois pontos e assim sucessivamente. Os pontos atribuídos pelos decisores a cada alternativa são somados e a alternativa que tiver obtido a menor pontuação é a escolhida. Todas as alternativas são ordenadas por ordem decrescente de pontuação, o que garante o respeito ao axioma da totalidade
(Arrow, 1951). No método de Condorcet também se exige que cada decisor ordene todas as alternativas de acordo com suas preferências. Porém, em vez de se atribuir uma pontuação a cada alternativa, o método estabelece relações de superação. Para cada par de alternativas verifica-se qual delas foi preferida pela maioria dos decisores. O método de Copeland é derivado do método de Condorcet e consiste em calcular a soma das vitórias menos as derrotas em uma votação por maioria simples. As alternativas são então ordenadas pelo resultado dessa soma. Este método tem a vantagem de fornecer uma ordenação total. Apesar de originalmente os métodos ordinais terem sido propostos para problemas multidecisor, foram aqui usados como multicritério. Isto significa que não houve um decisor para ordenar as alternativas: foi usada a ordenação de cada critério na solução do algoritmo de cada método.

\section{RESULTADOS E DISCUSSÃO}

Não houve interação significativa entre os sistemas de cultivo e cultivares de alface, bem como efeito significativo destes fatores tratamentos no indicador biológico Uso Eficiente da Terra (UET) e no indicador econômico Índice de Lucratividade (IL) (Tabela 1). Porém, verificou-se que os UETs dos diversos sistemas consorciados foram significativamente superiores ao índice do cultivo solteiro da cenoura (Tabela 1). Isso indica que nos sistemas consorciados ocorreu melhor aproveitamento dos recursos ambientais, quando comparados ao sistema solteiro. Segundo Jagannath \& Sunderaraj (1987), em qualquer comparação de benefícios entre sistemas consorciados com áreas de ocupação de terra diferentes, a vantagem da consorciação via UET, vem de duas fontes diferentes, geralmente, confundidas: (a) fator terra (área ocupada pelas culturas) e (b) fator biológico/ agronômico (advindo dos tratamentos testados). Nos sistemas de cultivo associados testados, essa vantagem no UET variou de $90 \%$ a $133 \%$ (Tabela 2) e adveio do fator biológico/agronômico decorrente da combinação dos sistemas de cultivo e cultivares testadas, já que a área ocupada por cada cultura nos sistemas foi a mesma.

Com relação ao índice de lucratividade não se observou diferença significativa entre o cultivo solteiro e o consorciado (Tabela 1). No entanto, pode-se observar pelos valores médios que as vantagens biológicas auferidas nos fatores-tratamentos foram confirmadas em termos de lucratividade, que variaram de cerca de $66 \%$ a $71 \%$ (Tabela 2). Segundo Beltrão et al. (1984), os agrossistemas consorciados devem ser julgados em relação ao monocultivo, através de indicadores econômicos e agronômicos. Por outro lado, Anandajayasekeram (1990) relata que indicadores econômicos podem guiar a decisões e recomendações, bem como seleção de tratamentos e níveis em estágio inicial de uma pesquisa em consórcio.

A eficiência produtiva foi calculada segundo um modelo DEA, com uso do software SIAD (Angulo Meza et al., 2005). O modelo de input único e unitário aqui proposto (cujos aspectos teóricos são analisados em Lovell \& Pastor, 1999) é equivalente ao modelo multicritério aditivo, com a particularidade de que as próprias alternativas atribuem pesos a cada critério, ignorando qualquer opinião de um eventual decisor. Ou seja, DEA é usado como ferramenta multicritério e não como medida de eficiência clássica. Nesse 
caso, ao invés de um esquema de pesos fixos para cada critério, este seria um método com pesos variáveis, atribuídos da forma mais benevolente a cada DMU. O emprego de DEA no contexto multicritério foi estudado, por exemplo, em Stewart (1996), Farinaccio \& Ostanello (1999) e Soares de Mello et al. (2002). Por outro lado, com o intuito de reduzir a benevolência dos modelos DEA clássicos, podem ser usados modelos DEA em que sejam incorporadas opiniões subjetivas dos decisores ou especialistas sobre importância das variáveis, por meio de restrições aos pesos, conforme apresentado em Allen et al. (1997).

Interação significativa entre sistemas de cultivo e cultivares de alface foi observada nas eficiências DEA (Tabela 1). Desdobrando a interação sistema de cultivo dentro de cada cultivar, observou-se que apenas o sistema consorciado em faixa de quatro fileiras de cenoura alternadas com quatro fileiras de alface teve eficiência produtiva significativamente superior a do sistema consorciado em faixa com três fileiras de cenoura alternadas com três fileiras de alface, dentro da cultivar Mesa 659. Por outro lado, desdobrando a interação cultivares de alface dentro de cada sistema de cultivo, observou-se que as cultivares de alface diferiram apenas dentro do sistema consorciado em faixa de quatro fileiras, com as cultivares Mesa 659 e Laurel sobressaindo em relação às demais (Tabela 1). Esta interação entre os fatores-tratamentos aconteceu presumivelmente porque na eficiência produtiva foram considerados não só aspectos de produtividade (vistos em parte pela UET), mas também aspectos econômicos, considerados em parte no IL. Dessa forma, os resultados desse modelo DEA trazem informações dos dois critérios anteriores, que isoladamente não foram constatados, mas que ao serem considerados conjuntamente fizeram com que os efeitos da interação fossem percebidos. Também foi observada uma eficiência produtiva significativamente superior nos sistemas consorciados em relação ao cultivo solteiro (Tabela 1). Este resultado vem corroborar com a afirmativa principal de que os sistemas consorciados têm sua produtividade biológica total superior à obtida

Tabela 2. Índice de uso eficiente da terra, índice de lucratividade, eficiência produtiva por DEA e ordenação obtida pelos métodos de Borda, Condorcet e Copeland, em sistemas consorciados de cenoura e alface americana, em dois sistemas de cultivo em faixas e de cenoura solteira (Land equivalent ratio (UET), profit margin (IL), yield efficiency by DEA and ranks obtained by Borda, Condorcet, and Copeland methods, in intercropping systems of carrot and crisp head lettuce cultivars, in two strip-intercropping systems and carrot in sole crop). Mossoró, UFERSA, 2002.

\begin{tabular}{lcccccc}
\hline Sistemas de cultivos * & UET & $\begin{array}{c}\text { IL } \\
\text { (\%) }\end{array}$ & $\begin{array}{c}\text { Eficiência } \\
\text { DEA }\end{array}$ & Borda & $\begin{array}{c}\text { Ranks } \\
\text { Condorcet }\end{array}$ & Copeland \\
\hline Cenoura + Lucy Brown (3F) & 2,26 & 68,0 & 0,752 & 5 & $3^{* *}$ & 5 \\
Cenoura + Lucy Brown (4F) & 2,17 & 66,1 & 0,751 & 6 & 4 & 6 \\
Cenoura + Tainá (3F) & 2,14 & 71,2 & 0,785 & 4 & $3^{* *}$ & 4 \\
Cenoura + Tainá (4F) & 1,90 & 66,0 & 0,663 & 7 & 5 & 7 \\
Cenoura + Laurel (3F) & 2,18 & 69,7 & 0,846 & 2 & 2 & 2 \\
Cenoura + Laurel (4F) & 2,15 & 68,0 & 0,856 & 5 & $3^{* *}$ & 5 \\
Cenoura + Mesa 659 (3F) & 2,33 & 69,5 & 0,756 & 2 & $3^{* *}$ & 3 \\
Cenoura + Mesa 659 (4F) & 2,30 & 67,6 & 0,998 & 1 & 1 & 1 \\
Cenoura Solteira & 1,00 & 71,7 & 0,971 & 3 & 1 & 2 \\
\hline
\end{tabular}

$* 3 \mathrm{~F}=$ Sistema de cultivo em faixa com três fileiras de cenoura alternadas com três fileiras de alface (Strip-intercropping system with three carrot rows alternated with three lettuce rows); 26

4F = Sistema de cultivo em faixa com quatro fileiras de cenoura alternadas com quatro fileiras de alface (Strip-intercropping system with four carrot rows alternated with four lettuce rows); **Ciclo de intransitividade (Intransitivity cycle).

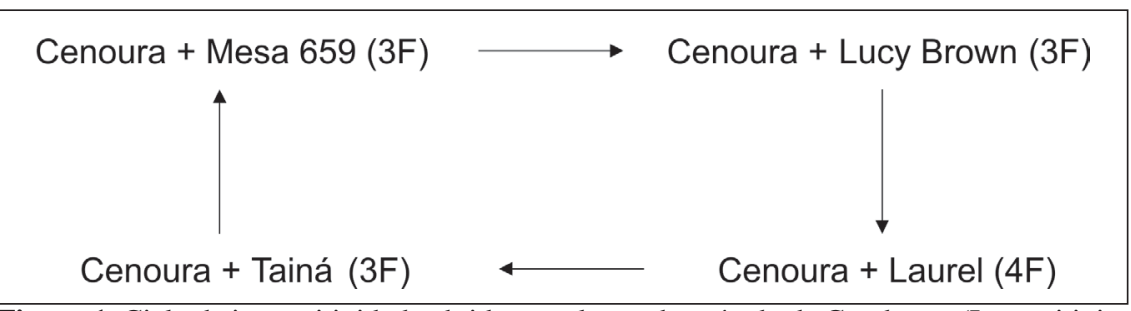

Figura 1. Ciclo de intransitividade obtido na solução do método de Condorcet (Intransitivity cycle obtained by Condorcet method). Mossoró, UFERSA, 2002.

nos cultivos solteiro das culturas (Park et al., 2002).

Pôde-se verificar que, apesar dos métodos Borda, Condorcet e Copeland ordenarem os sistemas de acordo com seu próprio algoritmo, os três apresentaram resultados semelhantes quanto aos melhores e piores sistemas de cultivo (Tabela 2). Pelos resultados desses métodos, que consideraram em seus algoritmos os três critérios (UET, IL e eficiência DEA), os sistemas de cultivo menos indicados são Cenoura + Tainá (4F) e Cenoura + Lucy Brown (4F). Sobressaíram-se como os melhores sistemas de cultivo Cenoura + Mesa 659 (4F), Cenoura + Laurel (3F) e Cenoura + Mesa 659 (3F). Apesar de originalmente os métodos ordinais terem sido propostos para problemas multidecisor, foram aqui usados como multicritério.
Isto significa que não houve um decisor para ordenar as alternativas: foi usada a ordenação de cada critério na solução do algoritmo de cada método. Cabe destacar que o método de Condorcet, considerado mais justo que o de Borda, tem a grande desvantagem de conduzir a situações de intransitividade, levando ao célebre "paradoxo de Condorcet". Este ocorre quando A é preferível a B, B é preferível a $\mathrm{C}$ e $\mathrm{C}$ é preferível a $\mathrm{A}$. $\mathrm{Na}$ solução do método de Condorcet para o caso aqui em estudo, por meio de destilações ascendentes e descendentes, não foi encontrada uma ordenação completa. Houve um empate e um ciclo de intransitividade (Figura 1), impedindo a hierarquização das alternativas constituintes do ciclo. Ressalta-se ainda que, quando há ciclos de intransitividade, o método de Copeland permite fazer a 
ordenação e mantém a classificação das alternativas que não pertencem a nenhum ciclo de intransitividade. Entretanto, assim como o método de Borda, o método de Copeland não respeita o axioma da independência em relação a alternativas irrelevantes de Arrow (1951), o que reforça o fato de não existir método multicritério "perfeito" (Arrow, 1951; Barba-Romero \& Pomerol, 1997).

Nesses resultados observa-se a importância de não considerar somente um aspecto na avaliação de consórcios. De fato, se fosse considerado somente o critério UET, o melhor sistema seria Cenoura + Mesa 659 (3F). Ao considerar somente o aspecto econômico (IL), surge como melhor sistema Cenoura Solteira seguido de Cenoura + Tainá (3F). Ao avaliar o índice DEA, Cenoura + Mesa 659 (4F) apresenta o melhor resultado. No geral, observou-se que o sistema de cultivo a ser indicado ao produtor, é o sistema Cenoura + Mesa 659 (4F), com altos indicadores agroeconômicos, maior eficiência produtiva DEA e melhores colocações nas ordenações dos métodos multicritério. Os indicadores de eficiência produtiva e as ordenações foram mais eficazes na discriminação tanto das melhores, como das piores performances dos sistemas de cultivos estudados, quando comparados com os indicadores agroeconômicos.

Modelos DEA avançados podem ser aplicados aos dados deste artigo. Por exemplo, caso o especialista julgue que as variáveis usadas no modelo DEA têm importância diferenciada no cálculo da medida de eficiência produtiva, esses julgamentos podem ser inserido no modelo. No cálculo do índice DEA podese adicionar, por exemplo, a restrição de que o output renda líquida tenha importância relativa maior que os outputs referentes às produtividades. Nesse caso, conforme anteriormente mencionado, devem ser usados modelos DEA com restrições aos pesos, que permitem inserir julgamentos de valor sobre a importância das variáveis no modelo.

\section{REFERÊNCIAS}

ALLEN R; ATHANASSOPOULOS A; DYSON RG; THANASSOULIS E. 1997. Weights restrictions and value judgments in data envelopment analysis: evolution, development, and future directions. Annals of Operations Research 73: 13-34.

ANANDAJAYASEKERAM P. 1990. Appropriate experimental designs and treatments structures for intercropping. In: WADDINGTON SR; PALMER AFE; EDJE OT. Research methods for cereal/legume intercropping. Proceedings... Lilongwe, Malawi: CIMMYT. p. 147-153.

ANGULO MEZA L; BIONDI NETO L; SOARES DE MELLO JCCB; GOMES EG. 2005. ISYDS - Integrated System for Decision Support (SIAD - Sistema Integrado de Apoio à Decisão): a software package for Data Envelopment Analysis model. Pesquisa Operacional 25: 493-503.

ARROW KJ. 1951. Social choice and individual values. New York: Wiley. 138p.

BARBA-ROMERO S; POMEROL JC. 1997. Decisiones multicriterio: fundamentos teóricos e utilización práctica. Madrid: Universidad de Alcalá. 420p.

BELTRÃO NEM; NÓBREGA LB; AZEVEDO DMP; VIEIRA DJ. 1984. Comparação entre indicadores agroeconômicos de avaliação de agroecossistemas consorciados e solteiros envolvendo algodão "upland" e feijão "caupi". Campina Grande: CNPA. 21p. (Boletim de Pesquisa 15).

CHARNES A; COOPER WW; RHODES E. 1978. Measuring the efficiency of decision-making units. European Journal of Operational Research 2: 429-444.

COOPER WW; SEIFORD LM; TONE K. 2000. Data Envelopment Analysis: a comprehensive text with models, applications, references and DEA-Solver software. Boston: Kluwer Academic Publishers. 318p.

DE KOEIJER TJ; WOSSINK GAA; STRUIK PC; RENKEMA JA. 2002. Measuring agricultural sustainability in terms of efficiency: the case of Dutch sugar beet growers. Journal of Environmental Management 66: 9-17.

EMPRESA BRASILEIRA DE PESQUISA AGROPECUÁRIA. Centro Nacional de Pesquisa de Solos. 1999. Sistema brasileiro de classificação de solos. Brasília: Embrapa Produção de Informação; Rio de Janeiro: Embrapa Solos. $412 \mathrm{p}$.

FARINACCIO F; OSTANELLO A. 1999. Evaluation of DEA validity as a MCDA/M tool: some problems and issues. Technical Report, TR-99-06. Itália: Dipartimento de Informatica, Università di Pisa.
GOMES EG; SOUZA GS. 2005. Avaliação de ensaios experimentais com o uso da análise de envoltória de dados: uma aplicação a consórcios. In: REUNIÃO ANUAL DA REGIÃO BRASILEIRA DA SOCIEDADE INTERNACIONAL DE BIOMETRIA (RBRAS), 50.; SIMPÓSIO DE ESTATÍSTICA APLICADA À EXPERIMENTAÇÃOAGRONÔMICA(SEAGRO), 11. Resumos... Londrina: IBS. 5p. (CD-ROM).

JAGANNATH MK; SUNDERARAJ N. 1987. Productivity equivalent ratio and statistical testing of its advantage in intercropping. Journal of the Indian Society of Agricultural Statistics 39: 289-300.

LETA FR; SOARES de MELLO JCCB; GOMES EG; ANGULO-MEZA L. 2005. Métodos de melhora de ordenação em DEA aplicados à avaliação estática de tornos mecânicos. Investigação Operacional 25: 229-242.

LOVELL CAK; PASTOR JT. 1999. Radial DEA models without inputs or without outputs. European Journal of Operational Research 118: 46-51.

MEAD R. 1990. Appropriate experimental designs and treatments structures for intercropping. In: WADDINGTON SR; PALMER AFE; EDJE OT. Research methods for cereal/legume intercropping. Proceedings... Lilongwe: CIMMYT. p. 131-138.

OLIVEIRA EQ. 2004. Desempenho agroeconômico do bicultivo de alface consorciada, em faixa, com cenoura. Mossoró: ESAM. 76p. (Tese mestrado).

PARK SE; BENJAMIN LR; WATKINSON AR. 2002. Comparing biological productivity in cropping systems: a competition approach. Journal of Applied Ecology 39: 416-426.

RILEY J. 1984. A general form of the land equivalent ratio. Experimental Agriculture 20: 19-29.

SEMENTES SAKAMA. 2002. Características de cultivares de alface. São Paulo: Sementes Sakama. 2p. (Mimeografado).

SILVA VF. 1999. Cultivares de alface em diferentes espaçamentos sob temperatura e luminosidade elevadas na região de Mossoró-RN. MossoróRN: ESAM. 25p. (Tese mestrado).

SIQUEIRA GAS. 1995. Espaçamentos de plantio na produção de cenoura "Brasília", no município de Mossoró-RN. Mossoró: ESAM. 23p. (Monografia Agronomia).

SOARES MELLO JCCB; GOMES EG. 2004. Eficiências aeroportuárias: uma abordagem comparativa com análise de envoltória de dados. Revista de Economia e Administração 3: 15-23.

SOARES MELLO JCCB; GOMES EG; SOARES MELLO MHC; LINS MPE. 2002. Método multicritério para seleção de variáveis em modelos DEA. Pesquisa Naval 15: 55-66.

STEWART TJ. 1996. Relationships between data envelopment analysis and multicriteria decision analysis. Journal of the Operational Research Society 47: 654-665.

VIEIRA JV; VECCHIA PTD; IKUTA H. 1983. Cenoura Brasília. Horticultura Brasileira 1: 42. 